\title{
Association between PPAP2B gene polymorphisms and coronary heart disease susceptibility in Chinese Han males and females
}

\author{
Yu-Xiao Sun ${ }^{1}$, Chuan-Yu Gao ${ }^{2}$, Yang Lu ${ }^{3}$, Xin Fu ${ }^{4}$, Jun-Ge Jia ${ }^{1}$, Yu-Jie Zhao ${ }^{1}$, Lian- \\ Dong Li ${ }^{1}$, Hong-zhi Dui ${ }^{1}$, Xing-Yu Zhang ${ }^{1}$, Zhi-Ying Li ${ }^{1}$, Lei Lei ${ }^{1}$, Wei-Feng Zhang ${ }^{1}$, \\ Yi-Qiang Yuan ${ }^{1}$ \\ ${ }^{1}$ Department of Cardiology, The Seventh People's Hospital, Zhengzhou, Henan, 450016, China \\ ${ }^{2}$ Department of Cardiology, Henan Provincial People's Hospital, Zhengzhou, 450000, China \\ ${ }^{3}$ Department of Medicine, Xi'an Jiaotong University, Xi'an, Shaanxi 710061, China \\ ${ }^{4}$ Department of Cardiology, The First Affiliated Hospital of Zhengzhou University, Zhengzhou 450052, China \\ Correspondence to: Yu-Xiao Sun, email: syxxjb@163.com \\ Yi-Qiang Yuan, email: yiqiangyuan@163.com \\ Keywords: coronary heart disease (CHD), single nucleotide polymorphisms (SNPS), PPAP2B, Chinese Han population, case-control study \\ Received: October 29, 2016 \\ Accepted: December 15, 2016 \\ Published: January 04, 2017
}

\section{ABSTRACT}

Little is known about gender-related differences in the association between PPAP2B single nucleotide polymorphisms (SNPs) and coronary heart disease (CHD) in Chinese Han males and females. We therefore conducted a case-control study with 456 cases and 685 healthy controls divided into male and female subgroups. Five PPAP2B polymorphisms (SNPs) were selected and genotyped using Sequenom Mass-ARRAY technology. Odds ratios (OR) and $95 \%$ confidence intervals (CIs) were calculated using unconditional logistic regression adjusting for age and gender. Allelic model analysis revealed that for PPAP2B rs1759752, allele frequency distributions differed between cases and controls in the male subgroup $(p=0.015$, OR: $1.401,95 \% C I$ : 1.066-1.481). Genetic model analysis revealed that in the male subgroup, rs 1759752 was associated with increased CHD risk in the dominant model $(p=0.035)$ and overdominant model $(p=0.045)$. In the female subgroup, rs12566304 was associated with a decreased CHD risk in the codominant model $(p=0.038)$ and overdominant model $(p=0.031)$. Additionally, the "GC" haplotypes of rs1759752 and rs1930760 were protective against CHD in males. These observations shed new light on genderrelated differences in the association between PPAP2B gene polymorphisms and CHD susceptibility in the Chinese Han population.

\section{INTRODUCTION}

Coronary heart disease (CHD) is a major cause of death and disability worldwide [1]. The incidence of CHD is higher in males than females. In China, moreover, the prevalence of CHD is increasing. One large study from the U.S. puts the annual mortality rate due to CHD there at 5\% among males and 3.65\% among females [2]. CHD is characterized by remodeling and narrowing of the coronary arteries, which supply blood, and thus oxygen, to the heart [3]. Twins study has shown that heritable factors account for $30-60 \%$ of interindividual variation in the risk of CHD [4], which means genetic factors are key to an individual's susceptibility to CHD.
Various genomic regions and gene polymorphisms have been identified as being associated with the risk of CHD [5-7]. To date, genome-wide association studies (GWAS) have identified more than 40 common variants associated with the risk of CHD $[8,9]$. For example, PPAP2B encodes lipid phosphate phosphatase 3 (LPP3), which is required for vascular development and may also contribute to the pathogenesis of human atherosclerotic disease [10]. A previous study showed that the rs 17114046 single nucleotide polymorphism (SNP) in the final intron of $P P A P 2 B$ is associated with an increased risk of CHD in Europeans and South Asians [11]. Another recent study observed that the CHD protective A allele of rs 72664324 at the $P P A P 2 B$ locus increases the transcriptional enhancer response to oxidized low-density lipoprotein 
in macrophages, which results in an alteration in LPP3 activity, and in turn promotes increased metabolism of proinflammatory mediators within atherosclerosis lesions [12].

The abovementioned studies suggest genetic polymorphisms in $P P A P 2 B$ are closely associated with the risk of CHD. On the other hand, little is known about the contribution of $P P A P 2 B$ SNPs to gender-related differences in CHD risk, especially in the Chinese Han population. We therefore performed a case-control study to investigate the associations between PPAP2B SNPs and the risk of CHD in Chinese Han males and females.

\section{RESULTS}

We recruited 456 cases ( 291 males and 165 females; average age at diagnosis: $59.56,64.01)$ and 685 controls (385 males and 300 females; average age: $47.55,49.93$ ) for this study. The characteristics of the cases and controls are shown in Table 1. We divided the samples into male and female subgroups, and multivariate analyses were adjusted for age.

A total of five $P P A P 2 B$ SNPs were identified in the cases and controls. All SNP call rates exceeded $98.2 \%$, which was considered high enough to perform association analyses. In the controls, all SNPs were in Hardy-Weinberg equilibrium (HWE) (Table 2). For SNP rs1759752, the allele frequency distributions differed between male cases and controls. ( $p=0.015$, OR: 1.401 , 95\%CI: 1.066-1.481), whereas there was no significant difference in the female subgroup. We also compared the genotype frequencies between cases and controls. For SNP rs1759752, the genotype frequency distributions also differed between the male cases and controls $(p=$ 0.019 ), while for rs 12566304 , the genotype frequency distributions differed between female cases and controls $(p=0.020)$ (Table 3).

We assumed the minor allele of each SNP was a CHD risk factor compared to the wild-type allele and analyzed associations between SNPs and CHD in various inheritance models (Table 4). In the male subgroup, rs1759752 was associated with an increased CHD risk in the dominant model $(p=0.035$, OR: 1.47 , 95\% CI: 1.03-2.11) and overdominant model ( $p=0.045$, OR: $1.46,95 \% \mathrm{CI}: 1.01-2.11)$. In the female subgroup, rs 12566304 was associated with a decreased CHD risk in the codominant model $(p=0.038$, OR: $0.57,95 \% \mathrm{CI}$ : $0.32-1.00)$ and overdominant model $(p=0.031$, OR: 0.54 , 95\% CI: 0.31-0.96).

The basic characteristics of the study subjects stratified by genotype are shown in Table 5. In the male subgroup, we found significant differences in the albumin (ALB), apolipoprotein A (aPOA1) and total protein (TP) concentrations, as well as the alanine aminotransferase (ALT)/aspartate aminotransferase (AST) ratio among differnet PPAP2B rs1759752 genotypes $(p<0.05)$. Subjects carrying the rs1759752 "CC" genotype had lower ALB, aPOA1 and TP concentrations and higher AST/ALT ratios than those carrying the "CG" and "GG" genotypes. In the female subgroup, we observed a significant difference in gamma-glutamyl transpeptidase (GGT) concentrations among the PPAP2B rs 12566304 genotypes $(p<0.05)$. Patients who carried the minor $\mathrm{C}$ allele displayed lower GGT levels than those with the wild-type A allele.

In the male subgroup, haplotype analysis revealed two blocks in PPAP2B (Figure 1): block 1 included rs1759752 and rs1930760, while block 2 included rs12566304 and rs914830. Further analyses of associations between $P P A P 2 B$ haplotypes and CHD risk showed that haplotype "GC" in block 1 was protective against CHD in males ( $p=0.03$, OR: $0.76,95 \%$ CI: 0.59 - 0.97) (Table 6).

\section{DISCUSSION}

In the present case-control study, we investigated the association between five PPAP2B SNPs and the risk of CHD in the Chinese Han population. We found that SNP rs1759752 is associated with an increased risk of CHD in males, while rs 12566304 is associated with a decreased risk of CHD in females. Additionally, we observed that haplotype "GC" of rs1759752 and rs1930760 was protective against $\mathrm{CHD}$ in males.

CHD was once considered as a cholesterol storage disease, but is now known to reflect complex interactions among molecular messages within the cells of the artery wall and in the blood [13]. Mammals express three lipid phosphate phosphatases (LPPs): LPP1, LPP2 and LPP3 encoded by $P P A P 2 A, P P A P 2 C$ and $P P A P 2 B$, respectively. In mice, loss of $P P A P 2 A$ [14] or inactivation of $P P A P 2 C$ [15] does not result in phenotypic alteration. However, $P P A P 2 B$ knockout is embryonically lethal, mainly due to defects in extra-embryonic vascular development [10], which suggests $P P A P 2 B$ plays an important role in vascular development.

$P P A P 2 B$ is located at locus $1 \mathrm{p} 32.2$. It is abundantly expressed in human plaque foam cells, and its specific enzymatic activity in macrophages was increased by oxidized low-density lipoprotein [12]. In our study, we found that males who carried the rs1759752 "CC" genotype had lower ALB, aPOA1 and TP concentrations and a higher AST/ALT ratio than those carrying the "CG" or "GG" genotype. In addition, female patients who carried the minor $\mathrm{C}$ allele of rs 12566304 displayed lower GGT levels than those carrying the wild-type A allele. It is noteworthy, that lower ALB, aPOA1, TP and GGT levels, which may reflect increased proteinase activity, and higher AST/ALT ratios are always associated with both liver and cardiovascular disease. We therefore suggest that $P P A P 2 B$ rs1759752 and rs12566304 SNPs may affect CHD risk by influencing LPP3 enzymatic activity. 
Table 1: Basic characteristics of CHD cases and healthy controls

\begin{tabular}{|c|c|c|c|c|c|c|}
\hline \multirow{2}{*}{ Variables } & \multicolumn{3}{|c|}{ Male } & \multicolumn{3}{|c|}{ Female } \\
\hline & Case & Control & $p$ & Case & Control & $p$ \\
\hline Number & 291 & 385 & & 165 & 300 & \\
\hline Age yr $($ mean \pm sd $)$ & $59.56 \pm 12.18$ & $47.55 \pm 10.655$ & $<0.001$ & $64.01 \pm 10.74$ & $49.93 \pm 7.743$ & $<0.001$ \\
\hline $\operatorname{ALB}(g / L)$ & $40.86 \pm 4.50$ & & & $41.13 \pm 0.30$ & & \\
\hline ALB/GLOB & $1.66 \pm 0.26$ & & & $1.59 \pm 0.01$ & & \\
\hline $\operatorname{ALT}(\mathrm{U} / \mathrm{L})$ & $32.32 \pm 34.17$ & & & $29.09 \pm 4.61$ & & \\
\hline AST (U/L) & $38.16 \pm 69.46$ & & & $33.32 \pm 3.49$ & & \\
\hline AST/ALT & $1.23 \pm 0.94$ & & & $1.42 \pm 0.08$ & & \\
\hline aPOA1 (g/L) & $1.22 \pm 0.24$ & & & $1.33 \pm 0.02$ & & \\
\hline aPOB $(g / L)$ & $0.97 \pm 0.37$ & & & $1.05 \pm 0.03$ & & \\
\hline aPOA1/aPOB & $1.45 \pm 0.71$ & & & $1.47 \pm 0.06$ & & \\
\hline $\mathrm{Ca}(\mathrm{mmol} / \mathrm{l})$ & $2.29 \pm 0.16$ & & & $2.29 \pm 0.01$ & & \\
\hline GGT (U/L) & $47.06 \pm 69.30$ & & & $40.39 \pm 7.46$ & & \\
\hline GLU (mmol/L) & $6.27 \pm 2.23$ & & & $6.45 \pm 0.21$ & & \\
\hline HDL-C (mmol/L) & $1.10 \pm 0.25$ & & & $1.19 \pm 0.02$ & & \\
\hline LDL-C (mmol/L) & $1.86 \pm 0.89$ & & & $2.02 \pm 0.57$ & & \\
\hline $\mathrm{Lp}(\mathrm{a})(\mathrm{mg} / \mathrm{L})$ & $227.16 \pm 253.31$ & & & $260.70 \pm 19.93$ & & \\
\hline MPV (fl) & $13.27 \pm 7.29$ & & & $12.75 \pm 0.59$ & & \\
\hline PLT (109/L) & $163.19 \pm 72.13$ & & & $180.38 \pm 0.29$ & & \\
\hline $\operatorname{PCT}(\%)$ & $1.25 \pm 3.45$ & & & $0.94 \pm 0.22$ & & \\
\hline PDW (\%) & $14.32 \pm 2.79$ & & & $14.07 \pm 0.26$ & & \\
\hline $\mathrm{TP}(\mathrm{g} / \mathrm{L})$ & $69.51 \pm 6.63$ & & & $67.229 \pm 0.455$ & & \\
\hline $\mathrm{TG}(\mathrm{mmol} / \mathrm{L})$ & $1.80 \pm 1.65$ & & & $1.80 \pm 0.10$ & & \\
\hline $\mathrm{TC}(\mathrm{mmol} / \mathrm{L})$ & $3.96 \pm 1.19$ & & & $4.33 \pm 0.09$ & & \\
\hline UA(umol/l) & $311.77 \pm 85.46$ & & & $265.80 \pm 6.24$ & & \\
\hline
\end{tabular}

ALB: albumin, ALB/GLOB: albumin/globulin, ALT: alanine aminotransferase, AST: aspartate aminotransferase, apoA: apolipoprotein A, apoB: apolipoprotein B, GGT: gamma-glutamyl transpeptidase, GLU: glucose, HDL: high-density lipoprotein, LDL: low-density lipoprotein, LP(a): lipoprotein, MPV: Mean Platelet Volume, PLT: platelet, PCT: plateletcrit, PDW: platelet distribution width, TP: total protein, TG: triglyceride, TC: total cholesterol, UA: Uric acid.

$p$ values were calculated using the Welch's test; $p<0.001$ indicates statistical significance.

Earlier GWAS studies revealed several common variants that influence CHD risk in populations of European ancestry. These include rs1333049, rs599839, rs3008621, rs501120, rs6922269 and rs2943634, among others [16]. Chinese researchers have replicated these studies to explore novel loci in the Chinese population, including rs6903956, rs2123536, rs1842896, rs9268402 and rs7136259 [17-19]. In the present study, we showed that rs1759752 is associated with increased CHD risk in males, while rs12566304 is associated with decreased CHD risk in females.

The present study has several potential limitations. First, the sample size is relatively small, and the participants included only Chinese population. Second, CHD is a very heterogeneous disease with many traditional risk factors, including tobacco use, physical inactivity, poor nutrition, obesity, hypertension, high blood cholesterol, and diabetes. We could not completely 
Table 2: Allele frequencies in cases and controls and odds ratio estimates for CHD

\begin{tabular}{|c|c|c|c|c|c|c|c|c|c|}
\hline \multirow{2}{*}{ SNP ID } & \multirow{2}{*}{ Gene(s) } & \multirow{2}{*}{ Band } & \multirow{2}{*}{ Alleles $\mathbf{A}^{\mathrm{a}} / \mathbf{B}$} & \multicolumn{2}{|c|}{ MAF } & \multirow{2}{*}{$\begin{array}{c}\text { HWE } \\
p\end{array}$} & \multirow{2}{*}{ ORs } & \multirow{2}{*}{ 95\% CI } & \multirow{2}{*}{$p$} \\
\hline & & & & Case & $\overline{\text { Control }}$ & & & & \\
\hline \multicolumn{10}{|l|}{ Male } \\
\hline rs11206831 & $P P A P 2 B$ & $1 \mathrm{p} 32.2$ & $\mathrm{~T} / \mathrm{C}$ & 0.050 & 0.055 & 1 & 0.909 & $0.559-1.478$ & 0.700 \\
\hline rs 1759752 & $P P A P 2 B$ & $1 \mathrm{p} 32.2$ & $\mathrm{C} / \mathrm{G}$ & 0.220 & 0.168 & 0.8558 & 1.401 & $1.066-1.841$ & $0.015^{*}$ \\
\hline rs 1930760 & $P P A P 2 B$ & $1 \mathrm{p} 32.2$ & $\mathrm{~T} / \mathrm{C}$ & 0.490 & 0.467 & 0.4733 & 1.093 & $0.881-1.356$ & 0.418 \\
\hline rs12566304 & $P P A P 2 B$ & $1 \mathrm{p} 32.2$ & $\mathrm{~A} / \mathrm{C}$ & 0.132 & 0.148 & 0.1561 & 0.877 & $0.643-1.198$ & 0.410 \\
\hline rs914830 & $P P A P 2 B$ & $1 \mathrm{p} 32.2$ & $\mathrm{~T} / \mathrm{C}$ & 0.324 & 0.329 & 0.8172 & 0.976 & $0.776-1.229$ & 0.838 \\
\hline \multicolumn{10}{|l|}{ Female } \\
\hline rs11206831 & $P P A P 2 B$ & $1 \mathrm{p} 32.2$ & $\mathrm{~T} / \mathrm{C}$ & 0.050 & 0.045 & 1 & 1.011 & $0.530-1.928$ & 0.975 \\
\hline rs1759752 & $P P A P 2 B$ & $1 \mathrm{p} 32.2$ & $\mathrm{C} / \mathrm{G}$ & 0.209 & 0.165 & 1 & 1.338 & $0.950-1.883$ & 0.094 \\
\hline rs 1930760 & $P P A P 2 B$ & $1 \mathrm{p} 32.2$ & $\mathrm{~T} / \mathrm{C}$ & 0.442 & 0.498 & 0.7299 & 0.799 & $0.610-1.046$ & 0.102 \\
\hline rs 12566304 & $P P A P 2 B$ & $1 \mathrm{p} 32.2$ & $\mathrm{~A} / \mathrm{C}$ & 0.161 & 0.148 & 0.1653 & 1.099 & $0.759-1.591$ & 0.619 \\
\hline rs914830 & $P P A P 2 B$ & $1 \mathrm{p} 32.2$ & $\mathrm{~T} / \mathrm{C}$ & 0.339 & 0.348 & 0.1621 & 0.961 & $0.724-1.246$ & 0.784 \\
\hline
\end{tabular}

MAF, minor allelic frequency; HWE, Hardy-Weinberg Equilibrium; ORs, odds ratios; CI: confidence interval.

${ }^{a}$ Minor allele; ${ }^{*} p \leq 0.05$ indicates statistical significance;

Table 3: Genotype distribution for SNPs in CHD patients and healthy controls

\begin{tabular}{|c|c|c|c|c|c|c|c|c|}
\hline \multirow{2}{*}{ SNP ID } & \multirow{2}{*}{ Alleles A $\mathbf{A}^{\mathrm{a} / B}$} & \multirow{2}{*}{ Genotype } & \multicolumn{2}{|c|}{ Male (No.) } & \multirow{2}{*}{$p^{\mathbf{b}}$} & \multicolumn{2}{|c|}{ Female (No.) } & \multirow{2}{*}{$p^{\mathrm{b}}$} \\
\hline & & & Case & Control & & Case & Control & \\
\hline \multirow[t]{3}{*}{ rs11206831 } & $\mathrm{T} / \mathrm{C}$ & $\mathrm{CC}$ & 262 & 344 & 0.942 & 150 & 273 & 1 \\
\hline & & $\mathrm{TC}$ & 29 & 40 & & 15 & 27 & \\
\hline & & $\mathrm{TT}$ & 0 & 1 & & 0 & 0 & \\
\hline \multirow[t]{3}{*}{ rs 1759752} & $\mathrm{C} / \mathrm{G}$ & GG & 172 & 205 & $0.019 *$ & 103 & 209 & 0.246 \\
\hline & & $\mathrm{CG}$ & 110 & 106 & & 55 & 83 & \\
\hline & & $\mathrm{CC}$ & 9 & 11 & & 7 & 8 & \\
\hline \multirow[t]{3}{*}{ rs1930760 } & $\mathrm{T} / \mathrm{C}$ & $\mathrm{CC}$ & 76 & 105 & 0.607 & 55 & 77 & 0.208 \\
\hline & & $\mathrm{TC}$ & 145 & 199 & & 74 & 147 & \\
\hline & & $\mathrm{TT}$ & 70 & 80 & & 36 & 76 & \\
\hline \multirow[t]{3}{*}{ rs 12566304} & $\mathrm{~A} / \mathrm{C}$ & $\mathrm{CC}$ & 223 & 283 & 0.624 & 120 & 214 & $0.020 *$ \\
\hline & & $\mathrm{AC}$ & 59 & 90 & & 37 & 83 & \\
\hline & & AA & 9 & 12 & & 8 & 3 & \\
\hline \multirow[t]{3}{*}{ rs914830 } & $\mathrm{T} / \mathrm{C}$ & $\mathrm{CC}$ & 130 & 174 & 0.765 & 69 & 133 & 0.240 \\
\hline & & $\mathrm{TC}$ & 132 & 167 & & 80 & 125 & \\
\hline & & $\mathrm{TT}$ & 28 & 43 & & 16 & 42 & \\
\hline
\end{tabular}

a Minor alleles, OR: Odds ratio, CI: Confidence interval.

b: $p$ values were calculated from Pearson's chi-square test adjusted for age.

$* p \leq 0.05$ indicates statistical significance. 
Table 4: Association between significant SNPs and CHD in multiple inheritance models (adjusted for age)

\begin{tabular}{|c|c|c|c|c|c|c|c|c|}
\hline SNP & Model & Genotype & Group $=$ case & Group $=$ control & OR $(95 \% \mathrm{CI})$ & $p$ & AIC & BIC \\
\hline \multirow{10}{*}{$\begin{array}{l}\text { rs1759752 } \\
\text { in Male }\end{array}$} & \multirow[t]{3}{*}{ Codominant } & $\mathrm{G} / \mathrm{G}$ & $172(59.1 \%)$ & $265(69.4 \%)$ & 1 & \multirow[t]{3}{*}{0.11} & \multirow[t]{3}{*}{760.6} & \multirow[t]{3}{*}{778.6} \\
\hline & & $\mathrm{G} / \mathrm{C}$ & $110(37.8 \%)$ & $106(27.8 \%)$ & $1.48(1.02-2.15)$ & & & \\
\hline & & $\mathrm{C} / \mathrm{C}$ & $9(3.1 \%)$ & $11(2.9 \%)$ & $1.40(0.51-3.79)$ & & & \\
\hline & \multirow[t]{2}{*}{ Dominant } & $\mathrm{G} / \mathrm{G}$ & $172(59.1 \%)$ & $265(69.4 \%)$ & 1 & \multirow[t]{2}{*}{$0.035 *$} & \multirow[t]{2}{*}{758.6} & \multirow[t]{2}{*}{772.2} \\
\hline & & $\mathrm{G} / \mathrm{C}-\mathrm{C} / \mathrm{C}$ & $119(40.9 \%)$ & $117(30.6 \%)$ & $1.47(1.03-2.11)$ & & & \\
\hline & \multirow[t]{2}{*}{ Recessive } & $\mathrm{G} / \mathrm{G}-\mathrm{G} / \mathrm{C}$ & $282(96.9 \%)$ & $371(97.1 \%)$ & 1 & \multirow[t]{2}{*}{0.69} & \multirow[t]{2}{*}{762.9} & \multirow[t]{2}{*}{776.4} \\
\hline & & $\mathrm{C} / \mathrm{C}$ & $9(3.1 \%)$ & $11(2.9 \%)$ & $1.22(0.45-3.30)$ & & & \\
\hline & \multirow[t]{2}{*}{ Overdominant } & $\mathrm{G} / \mathrm{G}-\mathrm{C} / \mathrm{C}$ & $181(62.2 \%)$ & $276(72.2 \%)$ & 1 & \multirow[t]{2}{*}{$0.045^{*}$} & \multirow[t]{2}{*}{759} & \multirow[t]{2}{*}{772.6} \\
\hline & & $\mathrm{G} / \mathrm{C}$ & $110(37.8 \%)$ & $106(27.8 \%)$ & $1.46(1.01-2.11)$ & & & \\
\hline & Log-additive & - & - & - & $1.37(1.00-1.88)$ & 0.049 & 759.2 & 772.7 \\
\hline \multirow{10}{*}{$\begin{array}{l}\text { rs } 12566304 \\
\text { in Female }\end{array}$} & \multirow[t]{3}{*}{ Codominant } & $\mathrm{C} / \mathrm{C}$ & $120(72.7 \%)$ & $214(71.3 \%)$ & 1 & \multirow[t]{3}{*}{$0.038 *$} & \multirow[t]{3}{*}{411.9} & \multirow[t]{3}{*}{428.5} \\
\hline & & $\mathrm{C} / \mathrm{A}$ & $37(22.4 \%)$ & $83(27.7 \%)$ & $0.57(0.32-1.00)$ & & & \\
\hline & & $\mathrm{A} / \mathrm{A}$ & $8(4.8 \%)$ & $3(1 \%)$ & $2.83(0.60-13.46)$ & & & \\
\hline & \multirow[t]{2}{*}{ Dominant } & $\mathrm{C} / \mathrm{C}$ & $120(72.7 \%)$ & $214(71.3 \%)$ & 1 & \multirow[t]{2}{*}{0.14} & \multirow[t]{2}{*}{414.2} & \multirow[t]{2}{*}{426.6} \\
\hline & & $\mathrm{C} / \mathrm{A}-\mathrm{A} / \mathrm{A}$ & $45(27.3 \%)$ & $86(28.7 \%)$ & $0.67(0.39-1.15)$ & & & \\
\hline & \multirow[t]{2}{*}{ Recessive } & $\mathrm{C} / \mathrm{C}-\mathrm{C} / \mathrm{A}$ & $157(95.2 \%)$ & $297(99 \%)$ & 1 & \multirow[t]{2}{*}{0.11} & \multirow[t]{2}{*}{413.9} & \multirow[t]{2}{*}{426.3} \\
\hline & & $\mathrm{A} / \mathrm{A}$ & $8(4.8 \%)$ & $3(1 \%)$ & $3.32(0.71-15.53)$ & & & \\
\hline & \multirow[t]{2}{*}{ Overdominant } & $\mathrm{C} / \mathrm{C}-\mathrm{A} / \mathrm{A}$ & $128(77.6 \%)$ & $217(72.3 \%)$ & 1 & \multirow[t]{2}{*}{$0.031 *$} & \multirow[t]{2}{*}{411.8} & \multirow[t]{2}{*}{424.2} \\
\hline & & $\mathrm{C} / \mathrm{A}$ & $37(22.4 \%)$ & $83(27.7 \%)$ & $0.54(0.31-0.96)$ & & & \\
\hline & Log-additive & - & - & - & $0.84(0.53-1.33)$ & 0.45 & 415.8 & 428.3 \\
\hline
\end{tabular}

ORs, odds ratios; CI: confidence interval; AIC: Akaike's Information criterion; BIC: Bayesian Information criterion. $* p \leq 0.05$ indicates statistical significance.

Table 5: Clinical and biochemical characteristics of participants, stratified based on $P P A P 2 B$ gene polymorphism

\begin{tabular}{|c|c|c|c|c|c|}
\hline \multirow[t]{2}{*}{ SNP } & \multirow[t]{2}{*}{ Characteristics } & \multicolumn{3}{|c|}{ Genotypes $($ mean \pm sd) } & \multirow[t]{2}{*}{$p$} \\
\hline & & $\mathrm{CC}$ & $\mathrm{CG}$ & GG & \\
\hline \multirow{4}{*}{$\begin{array}{l}\text { rs } 1759752 \\
\text { in Male }\end{array}$} & ALB & $37.14 \pm 5.63$ & $40.56 \pm 4.52$ & $41.26 \pm 4.35$ & 0.019 \\
\hline & AST/ALT & $1.87 \pm 1.77$ & $1.37 \pm 1.20$ & $1.11 \pm 0.63$ & 0.011 \\
\hline & aPOA1 & $1.02 \pm 0.16$ & $1.20 \pm 0.22$ & $1.24 \pm 0.25$ & 0.014 \\
\hline & $\mathrm{TP}$ & $60.22 \pm 8.33$ & $65.45 \pm 6.58$ & $66.53 \pm 6.42$ & 0.010 \\
\hline \multirow{2}{*}{$\begin{array}{l}\text { rs } 12566304 \\
\text { in Female }\end{array}$} & & $\mathrm{CC}$ & $\mathrm{AC}$ & $\mathrm{AA}$ & \\
\hline & GGT & $34.21 \pm 62.98$ & $33.83 \pm 36.38$ & $159.63 \pm 335.72$ & 0.001 \\
\hline
\end{tabular}

ALB: albumin, ALB/GLOB: albumin/globulin, ALT: alanine aminotransferase, AST: aspartate aminotransferase, apoA: apolipoprotein A, GGT: gamma-glutamyl transpeptidase, HDL: high-density lipoprotein, TP: total protein, UA: Uric acid. $p \leq 0.05$ indicates statistical significance. 
Table 6: $P P A P 2 B$ haplotype frequencies and the association with the risk of CHD in male case and control patients (adjusted for age)

\begin{tabular}{lccccccc}
\hline Block & SNP & Haplotypes & Freq-case & Freq-control & OR (95\% CI) & $\boldsymbol{p}$ \\
\hline & & GT & 0.484 & 0.464 & 1 & - & $0.03 *$ \\
1 & rs1759752|rs 1930760 & GC & 0.296 & 0.369 & $0.76(0.59-0.97)$ & 0.11 \\
\hline
\end{tabular}

${ }^{*} p \leq 0.05$ indicates statistical significance.

Table 7: Primers used for this study

\begin{tabular}{llll}
\hline SNP_ID & 1st_PCRP & 2st_PCRP & UEP_SEQ \\
\hline rs11206831 & ACGTTGGATGTGACCTCACTGAGTCTGAAC & ACGTTGGATGAGGCAGGAACTATGCCTGTG & ccTCCTCACACCTTTGCAAGT \\
rs1759752 & ACGTTGGATGTCAGGGAGTCGACAACACAG & ACGTTGGATGGGGTTAGGATTACAAGAAGC & tagtATTACAAGAAGCCAAGTCCG \\
rs1930760 & ACGTTGGATGGCACTGGCTCAAGTTAACTG & ACGTTGGATGTTTAAACTCCAGGCTATTGC & gaggTTGATGAAATTAGTTACCTAGC \\
rs12566304 & ACGTTGGATGGAATGTTGGCACTGACAATG & ACGTTGGATGTCTCCTTATTCCCATGTGAC & ggttaATGTGACTGAGGATGAAACA \\
rs914830 & ACGTTGGATGGGAGACGACTTTTACTGAGC & ACGTTGGATGTCTTGGCCTGTACTTGAGTG & gTGAGTGGGTCTGGGCGT
\end{tabular}

UEP: Unextended mini-sequencing primer.

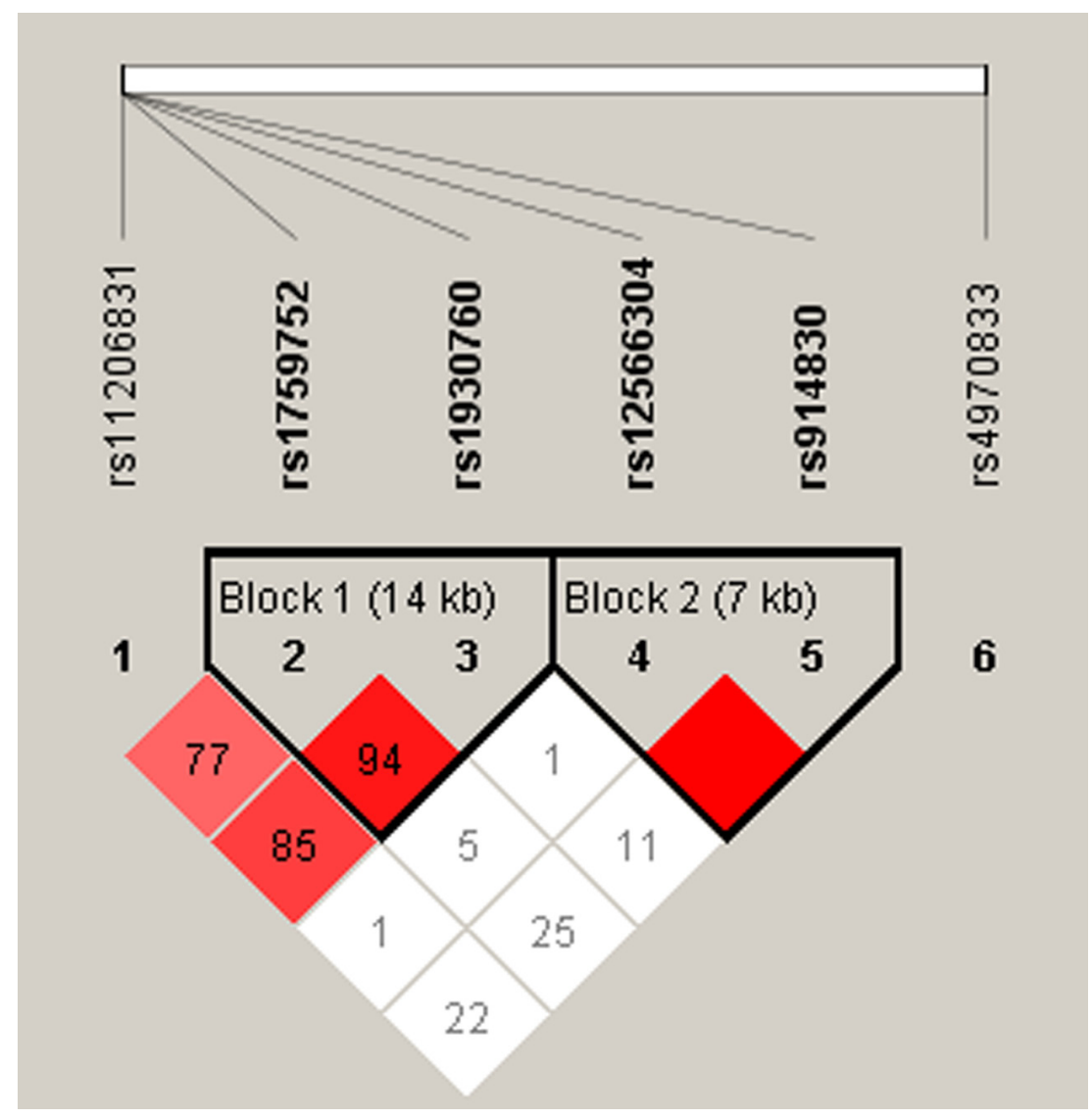

Figure 1: Haplotype block map for all the SNPs of the PPAP2B gene. 
eliminate the potential influences of these factors on the results.

In sum, our results indicate that $P P A P 2 B$ rs 1759752 is associated with an increased risk of CHD in males, while rs 12566304 was associated with a decreased risk of CHD in females. These SNPs may have the potentially to serve as prognostic biomarkers for CHD among the Chinese Han population. Further study will focus on determining the functional role of these SNPs and on validating our findings with a larger sample.

\section{MATERIALS AND METHODS}

\section{Ethics statement}

We strictly obeyed the World Medical Association Declaration of Helsinki when using human tissue and discussing the study protocol with subjects. The protocol was approved by the Ethical Committee of the Seventh People's Hospital of Zhengzhou. Each participant provided written, informed consent.

\section{Subjects}

All participants in our study were Han Chinese lived in Henan Province. A total of 456 CHD patients and 685 healthy controls were consecutively recruited between May 2013 and July 2015 in the Seventh People's Hospital of Zhengzhou, China. Patients were diagnosed with CHD using standard coronary angiography, which revealed $\geq 70 \%$ stenosis of the main branch of a coronary artery or aortic stenosis $\geq 50 \%$. Subjects with myocardial infarction, stable angina and unstable angina were classified as CHD subjects. There were no age, sex or disease-stage classification restrictions when enrolling the case group. Excluded were subjects with chronic diseases or conditions involving the brain, liver, heart or lung, and patients with more advanced cardiovascular, metabolic or endocrine diseases. Controls were healthy people receiving physical examinations in other clinical departments of Tangdu Hospital. Healthy controls did not have congenital heart disease, familial hypercholesterolemia, end-stage renal disease or vasculitis, which could have affected our study results. Peripheral blood was collected from both cases and controls for DNA extraction.

\section{Clinical data and patient demographics}

A standardized epidemiological questionnaire was provided to all subjects to collect basic demographic information, including sex, age, residence, educational status, history of family cancer, history of smoking and alcohol consumption. Plasma carcinoembryonic antigen and alpha-fetoprotein levels were determined to ensure that no controls suffered from any cancer.

\section{SNP selection and genotyping}

Candidate $P P A P 2 B$ SNPs were selected from previous publications that associated polymorphisms with CHD $[20,21]$. SNPs with minor allele frequencies (MAF) $>5 \%$ in the HapMap CHB population were selected. We validated five SNPs in PPAP2B. A GoldMag-Mini Purification Kit (GoldMag Co. Ltd. Xian city, China) was used to extract genomic DNA from whole blood samples. DNA concentrations were measured using a DU530 UV/ VIS spectrophotometer (Beckman Instruments, Fullerton, CA, USA). Using MassARRAY Assay Design 3.0 software (Sequenom, San Diego, CA, USA), we designed a multiplexed SNP MassEXTENDED assay [22]. SNPs were genotyped using the standard protocol recommended by the MassARRAY RS1000 (Sequenom) manufacturer, and data were analyzed using Typer 4.0 Software (Sequenom). The primers used for this study were listed in Table 7.

\section{Statistical analysis}

We used the Microsoft Excel and SPSS 17.0 (SPSS, Chicago, IL) statistical packages to perform the statistical analyses. All $p$ values were two-sided, and $p<$ 0.05 was considered statistically significant. A $t$ test and Chi-squared test were performed to compare sex and age differences between cases and controls. Fisher's exact test was applied to each SNP in the controls to test for departure from the Hardy-Weinberg Equilibrium (HWE). Odds ratios (ORs) and 95\% confidence intervals (CIs) for the allele and genotype frequencies were calculated using the Pearson Chi-square test adjusted for age (sex subgroups were separated) [23]. The Cochran-Armitage trend test was used to determine associations between SNPs and CHD.

PLINK software (http://pngu.mgh.harvard.edu/ purcell/plink/) was used to assess SNP associations with CHD risk in different genetic models (Codominant, dominant, recessive, overdominat and additive). We used unconditional logistic regression analysis to calculate ORs and 95\% CIs adjusted for age [24]. Pairwise linkage disequilibrium and haplotype constructions were performed using HAPLOVIEW 4.1 (http://broad.mit.edu/ mpg/haploview) [25].

\section{CONFLICTS OF INTEREST}

The authors have no conflicts of interest to report.

\section{REFERENCES}

1. Lopez AD, Mathers CD, Ezzati M, Jamison DT, Murray CJ. Global and regional burden of disease and risk factors, 
2001: systematic analysis of population health data. Lancet. 2006; 367:1747-1757.

2. Lerner DJ, Kannel WB. Patterns of coronary heart disease morbidity and mortality in the sexes: a 26-year follow-up of the Framingham population. Am Heart J. 1986; 111:383-390.

3. Sayols-Baixeras S, Lluis-Ganella C, Lucas G, Elosua R. Pathogenesis of coronary artery disease: focus on genetic risk factors and identification of genetic variants. The application of clinical genetics. 2014; 7:15-32.

4. Marenberg ME, Risch N, Berkman LF, Floderus B, de Faire U. Genetic susceptibility to death from coronary heart disease in a study of twins. The New England journal of medicine. 1994; 330:1041-1046.

5. Schunkert H, König IR, Kathiresan S, Reilly MP, Assimes TL, Holm H, Preuss M, Stewart AF, Barbalic M, Gieger C. Large-scale association analysis identifies 13 new susceptibility loci for coronary artery disease. Nature genetics. 2011; 43:333-338.

6. Deloukas P, Kanoni S, Willenborg C, Farrall M, Assimes TL, Thompson JR, Ingelsson E, Saleheen D, Erdmann J, Goldstein BA. Large-scale association analysis identifies new risk loci for coronary artery disease. Nature genetics. 2013; 45:25-33

7. Lu X, Wang L, Chen S, He L, Yang X, Shi Y, Cheng J, Zhang L, Gu CC, Huang J. Genome-wide association study in Han Chinese identifies four new susceptibility loci for coronary artery disease. Nature genetics. 2012; 44:890-894.

8. Peden JF, Farrall M. Thirty-five common variants for coronary artery disease: the fruits of much collaborative labour. Hum Mol Genet. 2011; 20:R198-205.

9. Deloukas P, Kanoni S, Willenborg C, Farrall M, Assimes TL, Thompson JR, Ingelsson E, Saleheen D, Erdmann J, Goldstein BA, Stirrups K, Konig IR, Cazier JB, et al. Large-scale association analysis identifies new risk loci for coronary artery disease. Nat Genet. 2013; 45:25-33.

10. Panchatcharam M, Salous AK, Brandon J, Miriyala S, Wheeler J, Patil P, Sunkara M, Morris AJ, Escalante-Alcalde D, Smyth SS. Mice with targeted inactivation of Ppap2b in endothelial and hematopoietic cells display enhanced vascular inflammation and permeability. Arteriosclerosis, thrombosis, and vascular biology. 2014; 34:837-845.

11. Consortium CADG. A genome-wide association study in Europeans and South Asians identifies five new loci for coronary artery disease. Nature genetics. 2011; 43:339-344.

12. Reschen ME, Gaulton KJ, Lin D, Soilleux EJ, Morris AJ, Smyth SS, O'Callaghan CA. Lipid-induced epigenomic changes in human macrophages identify a coronary artery disease-associated variant that regulates PPAP2B Expression through Altered C/EBP-beta binding. PLoS Genet. 2015; 11:e1005061.
13. Libby P, Theroux P. Pathophysiology of coronary artery disease. Circulation. 2005; 111:3481-3488.

14. Tomsig J, Snyder A, Berdyshev E, Skobeleva A, Mataya C, Natarajan V, Brindley D, Lynch K. Lipid phosphate phosphohydrolase type 1 (LPP1) degrades extracellular lysophosphatidic acid in vivo. Biochem J. 2009; 419:611-618.

15. Zhang N, Sundberg JP, Gridley T. Mice mutant for Ppap2c, a homolog of the germ cell migration regulator wunen, are viable and fertile. Genesis. 2000; 27:137-140.

16. Samani NJ, Deloukas P, Erdmann J, Hengstenberg C, Kuulasmaa K, McGinnis R, Schunkert H, Soranzo N, Thompson J, Tiret L, Ziegler A. Large scale association analysis of novel genetic loci for coronary artery disease. Arterioscler Thromb Vasc Biol. 2009; 29:774-780.

17. Tang NP, Wang LS, Yang L, Gu HJ, Sun QM, Cong RH, Zhou B, Zhu HJ, Wang B. Genetic variant in glutathione peroxidase 1 gene is associated with an increased risk of coronary artery disease in a Chinese population. Clinica chimica acta; international journal of clinical chemistry. 2008; 395:89-93.

18. Wang F, Xu CQ, He Q, Cai JP, Li XC, Wang D, Xiong X, Liao YH, Zeng QT, Yang YZ, Cheng X, Li C, Yang R, et al. Genome-wide association identifies a susceptibility locus for coronary artery disease in the Chinese Han population. Nat Genet. 2011; 43:345-349.

19. Lu X, Wang L, Chen S, He L, Yang X, Shi Y, Cheng J, Zhang L, Gu CC, Huang J, Wu T, Ma Y, Li J, et al. Genomewide association study in Han Chinese identifies four new susceptibility loci for coronary artery disease. Nat Genet. 2012; 44:890-894.

20. Dauriz M, Meigs JB. Current Insights into the Joint Genetic Basis of Type 2 Diabetes and Coronary Heart Disease. Current cardiovascular risk reports. 2014; 8:368.

21. Pechlivanis S, Muhleisen TW, Mohlenkamp S, Schadendorf D, Erbel R, Jockel KH, Hoffmann P, Nothen MM, Scherag A, Moebus S. Risk loci for coronary artery calcification replicated at 9p21 and 6q24 in the Heinz Nixdorf Recall Study. BMC medical genetics. 2013; 14:23.

22. Gabriel S, Ziaugra L, Tabbaa D. SNP genotyping using the Sequenom MassARRAY iPLEX platform. Current protocols in human genetics. 2009:2.12. 11-12.12. 16.

23. Adamec C. Example of the use of the nonparametric test. Test X2 for comparison of 2 independent examples. Ceskoslovenské zdravotnictví. 1964; 12:613.

24. Bland JM, Altman DG. Statistics notes. The odds ratio. BMJ (Clinical research ed). 2000; 320:1468.

25. Yong Y, Lin H. SHEsis, a powerful software platform for analyses of linkage disequilibrium, haplotype construction, and genetic association at polymorphism loci. Cell research. 2005; 15:97-98. 